\title{
Palliative Care for Neurologically Injured Patients: Why and How?
}

\author{
Barkha Bindu ${ }^{1} \quad$ Girija P. Rath ${ }^{1}$
}

Address for correspondence Girija P. Rath, MD, DM, Department of Neuroanaesthesiology and Critical Care, Neurosciences Centre, All India Institute of Medical Sciences (AlIMS), New Delhi 110029, India (e-mail: girijarath@yahoo.co.in).

\begin{abstract}
Keywords

- neuropalliative care

- end-of-life care

- do-not-resuscitate

- amyotrophic lateral sclerosis
\end{abstract}

The role of palliative care in patients with cancer is widely acknowledged and practiced. Though its role in chronic, progressive, and incurable neurological conditions is being recognized now, it is not widely practiced. Lack of awareness and training are important factors contributing to this. Neurological patients differ from oncological patients with respect to their symptoms, fluctuating disease course, varied prognoses, and difficulty in identifying end-of-life stage. Knowledge of different neurological conditions is important to practice neuropalliative care appropriately. Growth of neuropalliative care services must begin with the neurologist at the center of the multidisciplinary team. Neurologists need to be trained in communication skills and advance care planning, and must be aware of end-of-life care, do-not-resuscitate policies, and withdrawal of life support measures. This article elaborates on these aspects of neuropalliative care and discusses its role in various neurological conditions.

\section{Introduction}

The word "palliative" comes from the Latin word pallium meaning "to cloak," that is, alleviation of patient's symptoms. ${ }^{1}$ Earlier, palliative care (PC) was thought of as being a sequel to curative therapy and was restricted to the final stage of a disease. The World Health Organization (WHO) redefined PC in 1990 and integrated it into the treatment plan, irrespective of the disease outcome, and specified that patients and their families should receive PC early in the course of a disease. PC as defined by $\mathrm{WHO}$ is "an approach that improves quality of life of patients and their families facing the problems associated with life-threatening illness, through prevention and relief of suffering by means of early identification and impeccable assessment and treatment of pain and other problems, physical, psychosocial and spiritual." ${ }^{2}$ The new model of PC is often referred to as "simultaneous care," and it reiterates the need to integrate PC with standard care., ${ }^{3,4} \mathrm{PC}$ is now indicated right from the time of diagnosis of the incurable disease, all along the course of illness.

Palliative care services are broadly categorized as "basic" that is provided by all primary and secondary providers and "specialized" that is provided by PC specialists having appropriate education and training. ${ }^{5}$ The primary aim of PC is to reduce suffering of the patient by managing pain and other symptoms, and care of family and dependents, even after patients' death.

\section{Rationale Behind Extending Palliative Care to Neurological Patients}

Though the role of PC in cancer patients is widely acknowledged, it has been applied to other chronic illnesses such as end-stage renal disease, ${ }^{6}$ heart failure, ${ }^{7}$ and chronic pulmonary disease ${ }^{8}$ as well. There is growing awareness of its role in patients with chronic, progressive, disabling, and incurable neurological conditions. ${ }^{9}$

Palliative care specialists provide holistic care, including physical, psychosocial, and spiritual care, to oncological patients. Neurological patients have unique requirements owing to cognitive, mobility, communication, and other neurological impairments. PC can supplement traditional care of neurological patients in many ways, and it is an important component of care in this patient population.

\section{received}

August 30, 2018

accepted

September 24, 2018

published online

January 25, 2019
DOI https://doi.org/

$10.1055 / \mathrm{s}-0038-1675891$

ISSN 2348-0548.
Copyright $\odot 2019$ Indian Society of Neuroanaesthesiology and Critical Care
License terms

() (1) $\ominus \circledast$ 
First, traditional care of these patients aims at preserving functional capability and prolonging life, whereas PC aims at relieving suffering and looks at death as an expected outcome and not failure of treatment. ${ }^{10}$ Second, symptom burden in neurological patients has been shown to be similar to that in metastatic cancers. ${ }^{11}$ Lastly, even caregivers of neurological patients reportedly have similar rates of distress and burnout as caregivers of cancer patients. ${ }^{12}$

Currently, patients with neurological diseases are receiving "primary" PC, while "specialized" PC is lacking. Pain and feeding issues, common in oncological patients, may not be preeminent in neurological patients. "Specialized" PC including complex interventions is required in neurological patients to ensure that the services provided are not limited to end-of-life care (EOLC) and pain management. Some authors are even of the view that PC in neurology should be promoted as a subspecialty to cater to patients with prolonged and advanced neurological and neuromuscular diseases..$^{13} \mathrm{At}$ present, few neurologists have training even in primary PC. Several studies have shown that neurologists frequently fail to identify and treat symptoms, such as pain, depression, sleep, and fatigue, that influence quality of life of patients. ${ }^{14,15}$ The European Association of Palliative Care (EAPC) and European Academy of Neurology (EAN) recommend the PC team to be a multidisciplinary one, involving a physician, a nurse, and a social worker or a psychologist. ${ }^{16}$ Some authors suggest that the team must comprise a neurologist, a PC physician, a general practitioner, a physiatrist, a psychologist, a nurse, a social worker, and a patient care assistant. ${ }^{13}$

Neurological illnesses with fluctuating disease course such as motor neuron disease (MND), Parkinson's disease (PD), Alzheimer's disease, and multiple sclerosis (MS) often present with decline in functional status. These patients need PC in view of physical, psychological, cognitive, and psychiatric impairment. Stroke, despite being a major cause of death and disability, PC of these patients is still very limited. Unlike cancer patients, patients with acute stroke have greater functional impairment than psychological. Studies report that common reasons for availing PC services in stroke patients include dysphagia, nutrition, hydration and complex decision making. ${ }^{17}$ However, information is limited, and PC services for stroke patients need to be developed further. Traumatic brain injury (TBI) is gaining epidemic proportions across the world. While rehabilitation and PC are important needs of these patients, the availability of these services is hardly sufficient. PC has a crucial role in management of TBI patients with disabilities. Spinal cord compression due to metastases is a fairly common complication with some cancers. It commonly involves dorsal spine ${ }^{18}$ and presents with progressive paraplegia. Back pain, weakness of limbs, paraesthesia, numbness, and autonomic dysfunction are common symptoms. These patients suffer physical, emotional, and psychological distress making palliative and rehabilitative care essential components of patient care.

\section{Concept of Palliative Care: Neurological Perspective}

The concept of PC was originally developed based on the needs of cancer patients. There are major differences in the PC needs of patients with cancer and those with neurological illnesses. Neurological patients present with a wide range of symptoms that include marked cognitive, physical, and mental impairment, communication difficulties, variable duration and course of disease, and high incidence of associated comorbidities. Some diseases such as MND that are progressive and carry poor prognosis need PC early in the course of disease, whereas others such as PD generally need PC at later stages. Even psychosocial issues and caregiver needs vary from oncological patients. These aside, neurological illnesses often make it difficult to identify end-of-life stage. ${ }^{16,19}$ Survival sometimes far exceeds the anticipated life span, and at other times, some patients die abruptly due to complications. ${ }^{20}$ Another concern is the fact that a lot of neurological diseases set in at old age, demanding modification of drug dosages and knowledge of interactions between drugs and neurological status.

Since the course of the disease is unpredictable with most neurological illnesses, it is important to discuss patient's decisions early in the course of disease. ${ }^{21}$ There is immense variability in the prognosis of different neurological conditions. Conditions, such as severe stroke, severe brain injury, and aggressive neoplasms, have short survival periods, whereas progressive neurological conditions, such as PD, MS, movement disorders, and neuromuscular diseases, may have prolonged survival. While patients of amyotrophic lateral sclerosis (ALS) typically present when they are still cognitively intact, severe acute brain injuries generally catch patient and family unaware..$^{22}$

Palliative care needs of neurological patients vary over time resulting in an episodic nature of PC services. Important time points when PC services are usually required are at the time of diagnosis, disability onset, need for gastrostomy or tracheostomy, and at end of life. Common triggers that demand PC in patients with neurological illnesses include pain, neuropsychiatric symptoms, dysphasia, depression, and cognitive decline.

Palliative care services for neurological patient require multidisciplinary efforts by the physician, neurologist, and PC specialist. Apart from management of routine complaints, such as pain and constipation, that are common to cancer patients, issues such as spasticity, cognitive impairment, psycho-behavioral disorders, dysphagia, drooling, postural instability, and autonomic dysfunction need to be specifically addressed in neurological patients. PC specialists must also address family issues and provide physical, social, emotional spiritual and psychological care, and EOLC, as usual. PC services need to be provided throughout the illness and even after patients' death, in the form of bereavement support. The ultimate goal of PC is to improve the quality of life and reduce hospitalizations and suffering of the patient and family. 


\section{Amyotrophic Lateral Sclerosis/Motor Neuron Disease}

The earliest articles describing PC in neurology refer to patients of ALS. ${ }^{23}$ ALS is a lethal, neurodegenerative disorder. PC in these patients is recommended from the time of diagnosis. ${ }^{24}$ Progressive difficulty in breathing, swallowing and talking, and depression are common features of ALS. ${ }^{25}$ Dysphagia is the most common symptom, and nutrition management is an important component of ALS care. These patients have comparatively more psychological issues, including depression, hopelessness, suicidal tendency, and demoralization. ${ }^{26}$

The American Academy of Neurology (AAN) has published Practice Parameter Update for ALS care in 2009. This update supports specialized multidisciplinary clinic referrals and provides evidence-based recommendations on various aspects of ALS care such as management of medically refractory sialorrhea using botulinum toxin type B and low-dose radiation therapy, dextromethorphan/quinidine combination for pseudobulbar affect, and screening for cognitive and behavioral impairment. ${ }^{27}$

\section{Dementia}

Several symptoms in dementia require a palliative approach. Progressive cognitive disability, pain, agitation, feeding problems, comorbidities associated with old age, and communication difficulty are common issues. ${ }^{28}$ Behavioral disorders are the most difficult aspect to manage and require individualized approach. Judicious use of drugs to reduce agitation while avoiding excessive sleepiness at the same time and managing sleep problems while avoiding daytime somnolence mandate individualized management of behavioral disorders. Whether to perform invasive procedures or not in advanced dementia is an area of debate. Alleviation of suffering by symptomatic treatment must be the goal. ${ }^{29}$ Identification of end-of-life phase can be difficult in dementia. These patients may either reach terminal phase with severe physical impairment or die prematurely due to other reasons. Caregivers of dementia patients also have unique psychological issues and are prone to complicated bereavement. ${ }^{30}$

\section{Movement Disorders}

Typical manifestations of various movement disorders appear early in the course of these diseases. Early palliative approach is, thus, widely recommended in them. Patients with Huntington's disease have movement, psychiatric and cognitive issues, and require experienced caregivers for proper care. ${ }^{31}$ In late stages, movement disorders often show progressive motor, cognitive and behavioral deterioration, and side effects of long-term therapy.

Parkinson's disease (PD) is one of the most common neurodegenerative disorders occurring in elderly patients. ${ }^{32}$ It is characterized by postural instability, bradykinesia, and dystonia, often associated with pain. In early phase of the disease, treatment is generally targeted toward symptom relief, prevention of motor symptoms, and enhanced survival. In later stages, patient comfort and treatment of motor and nonmotor symptoms are the goals. Motor fluctuations (hypokinesia and hyperkinesia) significantly impair patient's quality of life. In end-stage PD, treatment aims at making the patient as independent as possible and for as long as possible. Nonmotor complications including cognitive impairment, sleep disturbances, autonomic dysfunction (orthostatic hypotension, bowel and bladder abnormalities, gastrointestinal disorders, sexual dysfunction), and neuropsychiatric disturbances (psychosis, depression, anxiety, apathy) tend to become more significant at the end stage and cause caregiver burden as well. Psychosis is treated by discontinuing or decreasing the offending drugs, which is usually one of the anti-PD drugs. ${ }^{33}$ Atypical antipsychotics might have to be added later. Depression and anxiety are common in late stages of PD and require careful evaluation. Serotonin reuptake inhibitors are commonly used for treatment of depression. ${ }^{34}$ Cognitive impairments are treated by discontinuing unnecessary psychotropic drugs and using acetyl cholinesterase inhibitors. Sleep disturbances can be managed by reducing anti-PD drugs and using lowdose clonazepam or melatonin at night time. ${ }^{35}$ Orthostatic hypotension, occurring in up to $20 \%$ of PD patients, is managed nonpharmacologically using compression stockings, increasing sodium intake, improving hydration, and raising head-end of bed slowly to let body's regulatory systems adapt. Pharmacological options include use of sympathomimetics, fludrocortisone, adrenergic agonist midodrine, and norepinephrine precursors such as droxidopa. ${ }^{36}$ Constipation is one of the most common gastrointestinal problems and is managed using laxatives, stool softeners, lactulose, increased water intake, and dietary bulk. Dysphagia occurring due to incoordination of the masticatory muscles, tongue, pharynx, and esophagus can lead to aspiration pneumonia, especially with liquids. Altered consistency of foods, safe swallowing techniques, and hand feeding can help. Rotigotine patch has recently been shown to be effective. ${ }^{37}$ Bladder dysfunction leading to nocturia, urgency, and increased urinary frequency occurs in up to $70 \%$ of patients. Use of toileting schedules, fluid restriction at evening, botulinum injections into prostate or urinary bladder wall, and cautious use of antimuscarinic agents can help manage urinary problems. Respiratory failure due to vocal cord adduction or hypoventilation can be managed by anti-PD medications, speech therapy, or noninvasive positive pressure ventilation. Pain in up to $50 \%$ of patients occurs commonly due to limb rigidity and dystonia. Adjustment of anti-PD drugs, simple analgesics, and adjuvants such as gabapentin usually suffices for pain management.

\section{Multiple Sclerosis}

Advanced stages of multiple sclerosis (MS) can be associated with significant disability. Cognitive impairment and multiorgan failure are frequent complications of MS. PC, in these patients, needs to target cognitive problems, feeding problems, bowel and bladder issues, psychiatric and sleep disorders, pain due to spasticity, and complications due to immobility such as pressure ulcers and infections. Communication and care of the caregivers are important aspects of PC in MS patients.

\section{Brain Tumors}

Brain tumors are different from other types of cancers in the extent of dependency they cause. Cognitive impairment, 
seizures, depression, communication problems, and psychiatric disorders that are commonly associated with brain tumors can cause significant deterioration of quality of life..$^{38}$ Some common issues that need to be kept in mind are side effects of steroid therapy and antiepileptic drugs. PC physicians must aim to optimize the preserved functional capability of these patients and improve quality of life, and not just EOLC.

Patients with malignant brain tumors, unlike general cancer patients, have disease specific symptoms in the end-of-life phase. In a study on patients with high-grade gliomas, loss of consciousness (87\%) and dysphagia (71\%) were the most common symptoms reported in the week before death. Other common symptoms reported at end of life were progressive neurological deficits, seizures, incontinence, headache, progressive cognitive deficits, and agitation/ confusion. Most common cause of death identified was brain herniation due to tumor progression. ${ }^{39}$

\section{Cerebrovascular Diseases}

Palliative care services must be individualized in these patients. The American Heart Association/American Stroke Association (AHA/ASA) has published Palliative and End-of-Life Care in Stroke scientific statement describing the basic principles of PC in stroke patients. ${ }^{40} \mathrm{~A}$ detailed discussion of this statement is beyond the scope of this review, but these guidelines emphasize the importance of integrating PC into general care of these patients. The major role of PC providers in these patients remains communicating with patient and family. Their preferences and needs, prognosis, death, choices regarding life-sustaining treatment, treatment options for various physical and psychological issues, rehabilitation options, etc. must be discussed.

A retrospective study on EOLC among cancer and stroke patients observed a higher prevalence of death rattles among stroke patients. The odds ratio of staff members not knowing about patient's symptoms, lack of communication, and lack of bereavement support were significantly higher among stroke patients compared with cancer patients. ${ }^{41}$ The ASA statement also recognizes the considerable unmet symptomatic needs in patients dying of stroke. It is suggested that using a specific symptom assessment checklist or the Palliative Care Needs Checklist may help better identify EOLC needs in stroke patients. ${ }^{42}$

\section{Traumatic Brain Injury}

Traumatic brain injury is gaining epidemic proportions worldwide and yields a huge patient population suffering with disabilities. PC in TBI has an important role to play in patients with impaired daily activities due to motor, sensory, and cognitive deficits, and in patients in vegetative state. Common complaints demanding PC services in TBI include motor deficits, spasticity, pain syndromes, and communication and psychological support to patient and family.

Palliative care must be initiated early in TBI patients as well. Common reasons reported for initiating PC in critically ill patients include patient characteristics (such as premorbid condition, age $>80$ years), selected diagnoses (such as global cerebral ischemia, multiple organ dysfunction), prolonged stay in intensive care unit (ICU), and tracheostomy or gastrostomy referrals. ${ }^{43}$ Identification of such triggers in TBI population may help in earlier involvement of PC specialists.

\section{Pediatric Neuropalliative Care}

Among pediatric patients, cerebral palsy, brain tumors, central nervous system malformations, inherited muscular dystrophies, and hypoxic ischemic encephalopathy are some common neurological conditions requiring $\mathrm{PC}^{44}$ Four categories of lifelimiting conditions in children that may benefit from PC have been identified by the Association of Children's Palliative Care. ${ }^{45}$ In children with neuromuscular conditions, four sequential phases in their life trajectories (peri-diagnostic phase, treatment decision-making phase, life-threatening events, terminal phase) have been recognized, and opportunities for integrating PC in each of these phases have been identified. ${ }^{46}$ In children with brain tumors, a parental survey revealed that EOL experience is characterized by the dying trajectory (neurological deterioration, loss of ability to communicate), parental struggles (competing responsibilities between parenting and work, difficult conversations about death with children), and barriers to dying at home (seizure control, financial and practical hardships of caring at home). ${ }^{47}$ Therefore, the needs of children are unique and different from adults receiving PC.

\section{Palliative Care Skills for Neurophysicians}

Familiarity with basic principles of PC is essential for all physicians treating neurological patients. This includes communicating bad news, management of nonmotor symptoms, advance care planning, and caregiver assessment. ${ }^{25}$

\section{Communicating Bad News}

Communication of diagnosis by physicians is often reported to be inadequate and ineffective, leaving patients with a feeling of dissatisfaction and loneliness. ${ }^{48}$

Some tools can be used while delivering bad news. The SPIKES tool (Setting up the interview, assessing patient's Perception, obtaining patient's Invitation, giving Knowledge, address Emotions, Strategy and Summary ${ }^{49}$ or the Vital Talk Web site ${ }^{50}$ can be used for help. Assessing patient's perception refers to finding out what the patient already knows about the diagnosis. Obtaining an invitation means finding out how much the patient wants to know. It should be done prior to revealing any distressing information to the patient. Once the patient seems willing to discuss his disease, a warning shot may be given to allow the patient some time to prepare for bad news. The diagnosis must then be revealed in clear terms and with empathy. Patient's emotions must be assessed and supported, if required. The patient must be given enough time to process the information and come out with any queries. It is a good practice to end the interview with a discussion regarding what to do and what to expect.

\section{Symptom Management}

Nonmotor symptoms are as important in neurological patients as they are in oncological patients, and should not 
be neglected. It is reported that up to $50 \% \mathrm{PD}$ patients with depression remain untreated. ${ }^{51}$ Pain, constipation, depression, dysphagia, and sleep issues are common nonmotor symptoms that limit functional capacity. Dysphagia is a common symptom, especially in ALS patients. Management options include altering dietary consistency, nutritional supplements, and alternative feeding methods such as percutaneous enteral gastrostomy (PEG). ${ }^{52}$ Muscle cramps are also seen in ALS patients, especially at night. Exercise and pharmacological therapy with baclofen and gabapentin can be used to treat painful cramps.

Delirium is a common symptom in stroke patients, more so in patients who are older and have preexisting cognitive impairment, severe stroke, and infection. Preventive measures include avoidance of sedative drugs, dehydration, and immobilization. Thinking and memory exercises, presence of family member, and maintaining regular circadian rhythm are helpful. Antipsychotic agents (avoiding benzodiazepines) may be used. Dexmedetomidine has been suggested as an option. ${ }^{53}$

Post-stroke depression is a fairly common entity and affects quality of life negatively. ${ }^{54}$ Regular screening and early psychotherapy ${ }^{55}$ with tricyclic antidepressants or selective serotonin reuptake inhibitors are reported to be useful.

\section{Advance Care Planning}

Advance care planning includes a discussion on living will, end-of-life, and CPR directives. It reduces chances of unnecessary aggressive treatment at end of life and must take place when the patient is still cognitively able. Health care professionals must have knowledge of prevailing policies in this context and must keep completed forms with patient records.

When discussed in advance, very low percentage of patients reportedly opt for tracheostomy and mechanical ventilation with disease progression, ${ }^{56}$ given the concerns regarding quality of life and when to remove patient from ventilator. However, if a patient opts for mechanical ventilation, clear criteria must be established regarding when to withdraw ventilator support.

\section{Caregiver Assessment}

The caregiver's quality of life has an impact on the patient's quality of life. Caregivers of terminally ill patients are at increased risk of depression, burnout, sleep problems, and even mortality. There is often a negative impact on the caregiver's social relationships, physical and psychological health, and finances, apart from the huge emotional impact. Needs of the caregiver have been identified as emotional support; referral to certain societies or associations that can provide caregivers with useful skills; and engaging in exercise, physical, and spiritual activities. ${ }^{57}$

Caregivers of ALS and MS patients have been found to have worsening of depression and increase in burden with physical decline of the patient. ${ }^{58,59}$ Physician must assess caregiver's ability to care for the patient and for themselves. Questions regarding the caregiver's health, emotional status, and their other obligations can give an idea about the caregiver's well-being. If the need is felt, neurologist may then refer the caregiver for counseling or other sources of support such as home health services. ${ }^{60}$

\section{End-of-Life Care}

One of the differences in PC services for oncological patients and neurological patients lies in identifying end-of-life stage. It is important to distinguish end-of-life phase from deterioration due to some other cause. Common criteria used to identify end of life in other patients include assisted ventilation and enteral nutrition via PEG but may not be indicative of the same in neurological patients. Common symptoms seen at end of life in neurological patients include swallowing problems, recurrent infection, weight loss, aspiration, physical status decline, cognitive deterioration, refusal to take feeds, and change in respiratory pattern. It is essential to recognize "triggers" that may indicate end of life to initiate $\mathrm{PC}$ at this stage.

A prior discussion must take place with family regarding resuscitation in the event of respiratory or cardiac arrest. This discussion is especially important in the context of patients who are not expected to benefit in any way from treatment except for mere survival. ${ }^{61}$

Withdrawal of nutrition and hydration is a controversial aspect owing to lack of well-defined guidelines. Whereas some consider it an assault to insert tubes into a person whose survival is uncertain, others consider that every life is precious and must receive maximum care at any stage. PC services can be helpful in decision making here. Both nutrition and hydration must be removed simultaneously. Death usually occurs in 10 to 14 days, peacefully. ${ }^{62}$

\section{Do-Not-Resuscitate Orders}

Do-not-resuscitate (DNR) recommendations were published in $1996^{63}$ and refer to withholding cardiopulmonary resuscitation (CPR). Outcome after CPR in patients with dementia and cognitive dysfunction is reportedly poorer when compared with cognitively intact individuals. ${ }^{64}$

It was proposed that two of the following criteria must be met before DNR orders ${ }^{63}$ :

- Severe stroke, defined as persistent deteriorating neurologic deficit, little or no activity on at least one side of body, and with impaired consciousness, global aphasia, or lack of response (Glasgow coma scale $[\mathrm{GCS}]<9$ ).

- Life-threatening brain damage, associated with brainstem compression, intraventricular extension, cerebellar lesions, infratentorial location involving multiple brainstem levels, or midline shift.

- Significant comorbidities, including pneumonia, pulmonary embolism, sepsis, recent myocardial infarction, cardiomyopathy, and life-threatening arrhythmias.

Palliative care team has an important role to play in explaining to both the treating physician and family members about DNR, and that it does not mean withdrawal of all aggressive therapy. 


\section{Withdrawal of Life Support Measures}

Decision of withdrawing life support measures in neurological patients is a difficult one as most of these patients are stable, survive long, and require only ventilatory support. The decision of "terminal extubation" should be taken only after proper consensus between the treating physician and family is reached. PC team plays an important role in decision making here; it has the duty of explaining the consequences of terminal extubation to the family along with managing post-extubation events. Survival time after extubation reportedly varies from 10 minutes to 11 days. ${ }^{65}$ Common complications encountered after terminal extubation include dyspnea, stridor, pain, death rattle, etc. PC team has the primary responsibility of easing the process of death, for both patient and family.

Opioids are commonly used for managing dyspnea and pain. Opioids reduce work of breathing and control tachypnea. Once symptoms settle, a continuous infusion is started and titrated. ${ }^{66} \mathrm{~A}$ common concern among physicians and families regarding use of opioids is the risk of respiratory depression that can hasten death. However, opioids actually reduce oxygen demand and improve tolerance to low oxygen levels. ${ }^{67}$

Stridor and "death rattle" can be distressing to the family. Stridor can be managed using steroids. "Death rattle" occurs due to excessive bronchopulmonary secretions and can be managed by reducing parenteral fluid intake and using diuretics in case of fluid overload and antisialagogues.

Propofol and benzodiazepines are commonly used agents for palliative sedation in patients with neurologic damage. Palliative sedation is required infrequently in these patients but may be required for relief of intractable symptoms that are not adequately managed with opioids. ${ }^{61}$

\section{The Dying Patient}

It is a common practice with health care providers to keep the dying patient away from family members and must be avoided. PC providers must provide physical, emotional, social, psychological, and spiritual care to the patient and family.

- Physical care of the dying patient involves withdrawing life support measures after discussion with family. All nonessential drugs including antibiotics and antacids are discontinued. Drugs that need to be continued, such as opioids, antiemetics, and anxiolytics may be converted to subcutaneous route, if feasible, and may be used as continuous infusions. Invasive interventions, such as intubation, central venous cannulation, blood tests, and invasive monitoring should be avoided. Good oral hygiene should be provided. Urinary catheter may be required for urinary retention. Symptoms, such as pain, vomiting, and agitation, must be adequately controlled. It is suggested that hydration and nutrition should be discontinued in most cases.$^{68} \mathrm{CPR}$ should be avoided in dying patients, and advanced directives of the patient must be respected. ${ }^{69}$
- Psychological care: Issues relating to death and dying must be dealt with sensitively. ${ }^{70}$ The patient should be helped to understand his/her condition and outcome.

- Social care: Family must be involved in understanding patient's condition and explained the fact that the patient will die. Family must be given time to stay with the patient, discuss patient's wishes, and say goodbye.

- Spiritual care: Patient's religious beliefs regarding how to deal with the body after death must be respected.

- Family issues: Interaction with family is an important component of PC services. It provides an opportunity to explain prognosis, set realistic expectations, and take prior decisions regarding EOLC. It also gives an opportunity to know the pending responsibilities of the dying person and help find the best way of finishing them. Family sessions expedite decision making and reduce suffering of both the patient and family.

Common questions faced in conditions such as ALS are regarding "how long" and expected quality of life, whereas in stroke patients, it is "how likely" are the chances of recovery. In immediate post-stroke period, family members need to take critical decisions such as intubation, mechanical ventilation, and lifesaving brain surgeries, that too rapidly. Consequently, family members pass through immense stress, fear, and anxiety. The PC physician has a responsibility to build trust, share information, and ease the process of decision making for the family. ${ }^{71}$

\section{Rehabilitation}

Rehabilitation services in PC setting aims at optimizing functional status of patients. It primarily involves the rehabilitation specialist, physiotherapist, occupational therapist, speech therapist, dietician, psychologist, nurses, general physicians, and spiritual preachers.

Rehabilitation services can be hospital based or home based. In the acute phase, hospital-based services must be provided. For chronic conditions, home-based rehabilitative care may be more affordable.

\section{Hospice for Neuropalliative Care}

Hospice is specialized care for patients in terminal stages of their illnesses, who are likely to die within 6 months. Guidelines are in place to determine which patients need hospice care. ${ }^{72}$ Though hospice services remain underutilized, they can provide optimal care even at patient's homes. It should be reinforced to the patient and family that hospice will provide holistic care, although it also confronts them with the fact that curative treatments are no longer working. ${ }^{73}$

\section{Conclusion}

It is well supported by evidence and is widely accepted that PC must be initiated early. There is now growing acknowledgment of the fact that PC has an important role to play in 
domains beyond oncology, especially in neurological illnesses as they cause significant disability. However, skills and knowledge pertaining to neurological pathologies is essential for practicing neuropalliative care.

A logical approach toward promoting neuropalliative care services would be to first train the physicians involved with neurologically impaired patients on basic PC. The physician must understand and decide when to involve other professionals. Integrating existing PC services for non-neurological conditions into neurological services might be the next step. It could be an integrative or consultative or mixed model of care delivery. The goal of neuropalliative care should be to provide counseling, manage patients' symptoms, and identify some "trigger points" that indicate clinical deterioration and predict end-of-life phase.

\section{Conflict of Interest}

None declared.

\section{References}

1 Muckaden M, Dighe M, Balaji P, et al. Paediatric palliative care: theory to practice. Indian J Palliat Care 2011;17 (Suppl):S52-S60

2 World Health Organization. Available at: www.who.int/cancer/palliative/definition. Accessed August 2, 2018

3 Meyers FJ, Linder J. Simultaneous care: disease treatment and palliative care throughout illness. J Clin Oncol 2003;21(7):1412-1415

4 Kelley AS, Meier DE. Palliative care-a shifting paradigm. N Engl J Med 2010;363(8):781-782

5 Ahmedzai SH, Costa A, Blengini C, et al; international working group convened by the European School of Oncology. A new international framework for palliative care. Eur J Cancer 2004;40(15):2192-2200

6 Hussain JA, Russon L. Supportive and palliative care for people with end-stage renal disease. $\mathrm{Br}$ J Hosp Med (Lond) 2012;73(11):640-644

7 Howlett JG. Palliative care in heart failure: addressing the largest care gap. Curr Opin Cardiol 2011;26(2):144-148

8 Heigener DF, Rabe KF. Palliative care concepts in respiratory disease. Respiration 2011;82(6):483-491

9 Hepgul N, Gao W, Evans CJ, et al; OPTCARE Neuro. Integrating palliative care into neurology services: what do the professionals say? BMJ Support Palliat Care 2018;8(1):41-44

10 Lanoix M. Palliative care and Parkinson's disease: managing the chronic-palliative interface. Chronic Illn 2009;5(1):46-55

11 Miyasaki JM, Long J, Mancini D, et al. Palliative care for advanced Parkinson disease: an interdisciplinary clinic and new scale, the ESAS-PD. Parkinsonism Relat Disord 2012;18(Supp 1)3: S6-S9

12 Kim Y, Schulz R. Family caregivers' strains: comparative analysis of cancer caregiving with dementia, diabetes, and frail elderly caregiving. J Aging Health 2008;20(5):483-503

13 Provinciali L, Carlini G, Tarquini D, Defanti CA, Veronese S, Pucci E. Need for palliative care for neurological diseases. Neurol Sci 2016;37(10):1581-1587

14 Inelmen EM, Mosele M, Sergi G, Toffanello ED, Coin A, Manzato E. Chronic pain in the elderly with advanced dementia. Are we doing our best for their suffering? Aging Clin Exp Res 2012;24(3):207-212

15 Attarian $\mathrm{H}$. Importance of sleep in the quality of life of multiple sclerosis patients: a long under-recognized issue. Sleep Med 2009;10(1):7-8
16 Oliver DJ, Borasio GD, Caraceni A, et al. A consensus review on the development of palliative care for patients with chronic and progressive neurological disease. Eur J Neurol 2016;23(1):30-38

17 Le BH, Pisasale M, Watt J. Palliative care in stroke. Palliat Med 2008;22(1):95-96

18 Loblaw DA, Perry J, Chambers A, Laperriere NJ. Systematic review of the diagnosis and management of malignant extradural spinal cord compression: the Cancer Care Ontario Practice Guidelines Initiative's Neuro-Oncology Disease Site Group. J Clin Oncol 2005;23(9):2028-2037

19 Dallara A, Tolchin DW. Emerging subspecialties in neurology: palliative care. Neurology 2014;82(7):640-642

20 Mitchell SL, Kiely DK, Hamel MB. Dying with advanced dementia in the nursing home. Arch Intern Med 2004;164(3):321-326

21 Voltz R, Akabayashi A, Reese C, Ohi G, Sass HM. End-of-life decisions and advance directives in palliative care: a cross-cultural survey of patients and health-care professionals. J Pain Symptom Manage 1998;16(3):153-162

22 Geurts M, Macleod MR, van Thiel GJ, van Gijn J, Kappelle LJ, van der Worp HB. End-of-life decisions in patients with severe acute brain injury. Lancet Neurol 2014;13(5):515-524

23 Kristjanson LJ, Nelson F, Henteleff P. Palliative care for individuals with amyotrophic lateral sclerosis. J Palliat Care $1987 ; 2(2): 28-34$

24 Oliver D, Borasio GD, Voltz R. The development of a consensus paper on palliative care in neurology: the implications for ALS care. Amyotroph Lateral Scler Frontotemporal Degener 2014;15:117-118

25 Boersma I, Miyasaki J, Kutner J, Kluger B. Palliative care and neurology: time for a paradigm shift. Neurology 2014;83(6):561-567

26 Clarke DM, McLeod JE, Smith GC, Trauer T, Kissane DW. A comparison of psychosocial and physical functioning in patients with motor neurone disease and metastatic cancer. J Palliat Care 2005;21(3):173-179

27 Miller RG, Jackson CE, Kasarskis EJ, et al; Quality Standards Subcommittee of the American Academy of Neurology. Practice parameter update: the care of the patient with amyotrophic lateral sclerosis: multidisciplinary care, symptom management, and cognitive/behavioral impairment (an evidence-based review): report of the Quality Standards Subcommittee of the American Academy of Neurology. Neurology 2009;73(15):1227-1233

28 Hughes JC, Jolley D, Jordan A, Sampson EL. Palliative care in dementia: issues and evidence. Adv Psychiatr Treat 2007; $13: 251-260$

29 Alzheimer Europe Report. End-of-life care for people with dementia. 2/2008. Available at: http://www.alzheimer-europe. org. Accessed August 2, 2018

30 Schulz R, Boerner K, Shear K, Zhang S, Gitlin LN. Predictors of complicated grief among dementia caregivers: a prospective study of bereavement. Am J Geriatr Psychiatry 2006;14(8):650-658

31 Dawson S, Kristjanson LJ, Toye CM, Flett P. Living with Huntington's disease: need for supportive care. Nurs Health Sci 2004;6(2):123-130

32 Checkoway H, Nelson LM. Epidemiologic approaches to the study of Parkinson's disease etiology. Epidemiology 1999;10(3):327-336

33 Friedman JH, Factor SA. Atypical antipsychotics in the treatment of drug-induced psychosis in Parkinson's disease. Mov Disord 2000;15(2):201-211

34 Weintraub D, Stern MB. Psychiatric complications in Parkinson disease. Am J Geriatr Psychiatry 2005;13(10):844-851 
35 Wilcox SK. Extending palliative care to patients with Parkinson's disease. Br J Hosp Med (Lond) 2010;71(1):26-30

36 Mostile G, Jankovic J. Treatment of dysautonomia associated with Parkinson's disease. Parkinsonism Relat Disord 2009;15(Suppl 3):S224-S232

37 Kenney C, Jankovic J. Rotigotine transdermal patch in the treatment of Parkinson's disease and restless legs syndrome. Expert Opin Pharmacother 2007;8(9):1329-1335

38 Schubart JR, Kinzie MB, Farace E. Caring for the brain tumor patient: family caregiver burden and unmet needs. Neuro-oncol 2008;10(1):61-72

39 Sizoo EM, Braam L, Postma TJ, et al. Symptoms and problems in the end-of-life phase of high-grade glioma patients. Neuro-oncol 2010;12(11):1162-1166

40 Holloway RG, Arnold RM, Creutzfeldt CJ, et al; American Heart Association Stroke Council, Council on Cardiovascular and Stroke Nursing, and Council on Clinical Cardiology. Palliative and end-of-life care in stroke: a statement for healthcare professionals from the American Heart Association/American Stroke Association. Stroke 2014;45(6):1887-1916

41 Eriksson H, Milberg A, Hjelm K, Friedrichsen M. End of life care for patients dying of stroke: a comparative registry study of stroke and cancer. PLoS One 2016;11(2):e0147694

42 Creutzfeldt CJ, Holloway RG, Curtis JR. Palliative care: a core competency for stroke neurologists. Stroke 2015; 46:2714-2719

43 Norton SA, Hogan LA, Holloway RG, Temkin-Greener H, Buckley MJ, Quill TE. Proactive palliative care in the medical intensive care unit: effects on length of stay for selected high-risk patients. Crit Care Med 2007;35(6):1530-1535

44 Medina RC, Cantero MJP, Rodriguez AM, et al. Palliative care and paediatric neurology. Eur J Paediatr Neurol 2015;19:S127

45 Johnson M. A guide to the development of children's palliative care services. Association of Children's Palliative Care (ACT). 3rd ed. Bristol, UK: Royal College of Nursing and Royal college of Paediatrics and Child Health; 2009

46 Rushton CH, Erby LH, Cohn RD, Geller G. Integrating palliative care in life-limiting pediatric neuromuscular conditions: the case of SMA-Type 1 and Duchene muscular dystrophy. J Palliat Care Med 2012;2:103

47 Zelcer S, Cataudella D, Cairney AE, Bannister SL. Palliative care of children with brain tumors: a parental perspective. Arch Pediatr Adolesc Med 2010;164(3):225-230

48 O'Brien MR, Whitehead B, Jack BA, Mitchell JD. From symptom onset to a diagnosis of amyotrophic lateral sclerosis/motor neuron disease (ALS/MND): experiences of people with ALS/ MND and family carers-a qualitative study. Amyotroph Lateral Scler 2011;12(2):97-104

49 Baile WF, Buckman R, Lenzi R, Glober G, Beale EA, Kudelka AP. SPIKES-A six-step protocol for delivering bad news: application to the patient with cancer. Oncologist 2000;5(4):302-311

50 VITAL talk. Available at: http://www.vitaltalk.org. Accessed August 8, 2018

51 Chen JJ, Marsh L. Depression in Parkinson's disease: identification and management. Pharmacotherapy 2013;33 (9):972-983

52 Miller RG, Jackson CE, Kasarskis EJ, et al; Quality Standards Subcommittee of the American Academy of Neurology. Practice parameter update: the care of the patient with amyotrophic lateral sclerosis: drug, nutritional, and respiratory therapies (an evidence-based review): report of the Quality Standards Subcommittee of the American Academy of Neurology. Neurology 2009;73(15):1218-1226

53 Mo Y, Zimmermann AE. Role of dexmedetomidine for the prevention and treatment of delirium in intensive care unit patients. Ann Pharmacother 2013;47(6):869-876
54 Hackett ML, Anderson CS, House A, Xia J. Interventions for treating depression after stroke. Cochrane Database Syst Rev 2008;(4):CD003437

55 Hackett ML, Anderson CS, House A, Halteh C. Interventions for preventing depression after stroke. Cochrane Database Syst Rev 2008;(3):CD003689

56 Albert SM, Murphy PL, Del Bene ML, Rowland LP. Prospective study of palliative care in ALS: choice, timing, outcomes. J Neurol Sci 1999;169(1-2):108-113

57 Sullivan AB, Miller D. Who is taking care of the caregiver? J Patient Exp 2015;2(1):7-12

58 Gauthier A, Vignola A, Calvo A, et al. A longitudinal study on quality of life and depression in ALS patient-caregiver couples. Neurology 2007;68(12):923-926

59 Ozmen S, Yurttas A. Determination of care burden of caregivers of patients with multiple sclerosis in Turkey. Behav Neurol 2018:7205046. eCollection 2018

60 Labson MC, Sacco MM, Weissman DE, Gornet B, Stuart B. Innovative models of home-based palliative care. Cleve Clin J Med 201380 Electronic(Suppl 1):eS30-eS35

61 Baker R, Wu AW, Teno JM, et al. Family satisfaction with endof-life care in seriously ill hospitalized adults. J Am Geriatr Soc 2000;48(5 Suppl):S61-S69

62 Alexandrov AV, Pullicino PM, Meslin EM, Norris JW. Agreement on disease-specific criteria for do-not-resuscitate orders in acute stroke. Members of the Canadian and Western New York Stroke Consortiums. Stroke 1996;27(2):232-237

63 Mayer SA, Kossoff SB. Withdrawal of life support in the neurological intensive care unit. Neurology 1999;52(8):1602-1609

64 Keenan SP, Busche KD, Chen LM, McCarthy L, Inman KJ, Sibbald WJ. A retrospective review of a large cohort of patients undergoing the process of withholding or withdrawal of life support. Crit Care Med 1997;25(8):1324-1331

65 Ebell MH, Becker LA, Barry HC, Hagen M. Survival after in-hospital cardiopulmonary resuscitation. A meta-analysis. J Gen Intern Med 1998;13(12):805-816

66 Bakker J, Jansen TC, Lima A, Kompanje EJ. Why opioids and sedatives may prolong life rather than hasten death after ventilator withdrawal in critically ill patients. Am J Hosp Palliat Care 2008;25(2):152-154

67 Chan JD, Treece PD, Engelberg RA, et al. Narcotic and benzodiazepine use after withdrawal of life support: association with time to death? Chest 2004;126(1):286-293

68 Joint Working Party between the National Council for Hospice and Specialist Palliative Care Services and the Ethics Committee of the Association for Palliative Medicine of Great Britain and Ireland. Ethical decision making in palliative care: artificial hydration for people who are terminally ill. J Eur Assoc Palliative Care 1997;4:203-207

69 British Medical Association. Advance Statements about Medical Treatment: Code of Practice with Explanatory Notes. London, UK: BMJ Publishing Group; 1995

70 Fallowfield LJ, Jenkins VA, Beveridge HA. Truth may hurt but deceit hurts more: communication in palliative care. Palliat Med 2002;16(4):297-303

71 Epstein RM, Street RL Jr. Shared mind: communication, decision making, and autonomy in serious illness. Ann Fam Med 2011;9(5):454-461

72 Mitsumoto H, Rabkin JG. Palliative care for patients with amyotrophic lateral sclerosis: "prepare for the worst and hope for the best." JAMA 2007;298(2):207-216

73 Casarett DJ, Quill TE. "I'm not ready for hospice": strategies for timely and effective hospice discussions. Ann Intern Med 2007;146(6):443-449 